Bull. Austral. Math. Soc.

VoL. 45 (1992) [479-482]

\title{
ON THE RATE OF CONVERGENCE IN THE STRONG LAW OF LARGE NUMBERS FOR ARRAYS
}

\author{
Tien-Chung Hu and N.C. Weber
}

\begin{abstract}
For sequences of independent and identically distributed random variables it is well known that the existence of the second moment implies the law of the iterated logarithm. We show that the law of the iterated logarithm does not extend to arrays of independent and identically distributed random variables and we develop an analogous rate result for such arrays under finite fourth moments.
\end{abstract}

\section{INTRODUCTION}

In traditional probability theory, the strong limit laws refer to those theorems which deal with almost sure convergence of a sequence of random variables. Let $\left\{X_{n}\right\}$ be a sequence of independent, identically distributed random variables and let $S_{n}=\sum_{k=1}^{n} X_{k}$, $n=1,2, \cdots$. Kolmogorov [3] proved the following result, known as the strong law of large numbers:

$$
\frac{1}{n} S_{n} \rightarrow E X_{1} \text { a.s. as } n \rightarrow \infty \text { if and only if } E\left|X_{1}\right|<\infty
$$

If the $\left\{X_{n}\right\}$ are assumed, in addition, to satisfy the conditions $E X_{n}=0$ and $E X_{n}^{2}<\infty$ for all $n \geqslant 1$, Hartman and Wintner [1] proved another fundamental result, known as the law of the iterated logarithm:

$$
P\left\{\limsup _{n \rightarrow \infty} \frac{S_{n}}{\sqrt{2 s_{n}^{2} \log \log s_{n}^{2}}}=1\right\}=1,
$$

where $s_{n}^{2}=\operatorname{Var}\left(S_{n}\right)$.

However, many important statistical problems are concerned with arrays of random variables, thus it is also natural to consider the strong limit laws for arrays of

Received 11 June 1991

Work was completed while the first author was visiting the Department of Statistics, University of North Carolina, Chapel Hill, and while the second author was visiting the Department of Statistics, University of British Columbia.

Copyright Clearance Centre, Inc. Serial-fee code: 0004-9729/92 \$A2.00+0.00. 
random variables. Let $\left\{X_{n k}\right\}$ be an array of independent, identically distributed random variables with $E X_{n k}=0$ for all $n$ and $k$. Let $S_{n}=\sum_{k=1}^{n} X_{n k}$ for $n=1,2, \cdots$. $\mathrm{Hu}$, Moricz and Taylor [2] obtained the strong law of large numbers for arrays $\left\{X_{n k}\right\}$. They showed that

$$
\frac{1}{n} \sum_{k=1}^{n} X_{n k} \rightarrow E X_{11} \text { a.s. as } n \rightarrow \infty \text { if and only if } E X_{11}^{2}<\infty \text {. }
$$

Thus to extend the strong law of large numbers from sequences to arrays we have to strengthen the finite first moment condition to finite second moments. We may now ask if the law of the iterated logarithm holds for arrays of random variables if the $r$ th moment is finite for some $r>2$. In this note we show that the law of the iterated logarithm does not hold for the arrays. Moreover, by applying results on the probabilities of moderate deviations we prove that if $E X_{11}^{4}<\infty$ then

$$
\limsup _{n \rightarrow \infty} \frac{S_{n}}{\sqrt{2 s_{n}^{2} \log s_{n}^{2}}}=1 \text { a.s., }
$$

where $s_{n}^{2}=\operatorname{Var}\left(S_{n}\right)$.

\section{MAIN RESULTS}

Let $\left\{X_{n k}\right\}$ be an array of independent and identically distributed random variables with $E X_{n k}=0$ and $E X_{n k}^{2}=1$, for all $n$ and $k$. In this case $\operatorname{Var}\left(S_{n}\right)=n$. We can now state our main result.

THEOREM 1. Let $\left\{X_{n k}\right\}$ be an array of independent and identically distributed random variables with mean 0 and variance 1 . If $E X_{11}^{4}<\infty$ then

$$
P\left(\limsup _{n \rightarrow \infty} \frac{S_{n}}{\sqrt{2 n \log n}}=1\right)=1 .
$$

PROOF: It suffices to show that for every $\varepsilon>0$,

$$
\begin{aligned}
& P\left(\frac{S_{n}}{\sqrt{2 n \log n}}>1+\varepsilon \text { i.o. }\right)=0, \\
& P\left(\frac{S_{n}}{\sqrt{2 n \log n}}>1-\varepsilon \text { i.o. }\right)=1,
\end{aligned}
$$

and

which, by the Borel Cantelli lemma, are respectively equivalent to

$$
\sum_{n=1}^{\infty} P\left(S_{n}>(1+\varepsilon) \sqrt{2 n \log n}\right)<\infty
$$

and

$$
\sum_{n=1}^{\infty} P\left(S_{n}>(1-\varepsilon) \sqrt{2 n \log n}\right)=\infty
$$


From Theorem 1 of Rubin and Sethuraman [5], if $E X_{11}^{4}<\infty$ then

$$
P\left(S_{n}>(1-\varepsilon) \sqrt{2 n \log n}\right) \sim[2(1-\varepsilon) \sqrt{\pi \log n}]^{-1} n^{-(1-\varepsilon)^{2}},
$$

and so (6) holds.

From Theorem 2 of Michel [4], with $c_{0}^{2}=2$, we have that $E X_{11}^{4}<\infty$ if and only if

$$
\sum_{n=1}^{\infty}(\log n)^{2} P\left(\left|S_{n}\right|>c \sqrt{n \log n}\right)<\infty, \text { for all } c>\sqrt{2}
$$

Thus

$$
\sum_{n=1}^{\infty}(\log n)^{2} P\left(S_{n}>(1+\varepsilon) \sqrt{2 n \log n}\right)<\infty, \text { for all } \varepsilon>0
$$

and so (5) holds.

From the theorem we can easily see that

$$
\limsup _{n \rightarrow \infty} \frac{S_{n}}{\sqrt{2 n \log \log n}}=\infty \text { a.s., }
$$

so the law of the iterated logarithm cannot hold in general for arrays of rowwise independent random variables. Further, we have the following partial converse to Theorem 1.

Theorem 2. Given $\left\{X_{n k}\right\}$ as above with $E\left|X_{11}\right|^{q}=\infty$, for some $q, 2<q<4$, then

$$
P\left(\limsup _{n \rightarrow \infty} \frac{S_{n}}{\sqrt{n \log n}}=\infty\right)=1
$$

Proof: The result will follow if, for all $c>0$,

$$
\sum_{n=1}^{\infty} P\left(S_{n}>c \sqrt{n \log n}\right)<\infty .
$$

Suppose that there is some $\eta>0$ such that

$$
\sum_{n=1}^{\infty} P\left(S_{n}>\eta \sqrt{n \log n}\right)<\infty
$$

Then $P\left(S_{n}>\eta \sqrt{n \log n}\right) \leqslant K n^{-1}$ for some $K>0$, and so from Theorem 5 of Rubin and Sethuraman [5], $E\left|X_{11}\right|^{q}<\infty$ for all $q<4$. This is a contradiction so we conclude that (8) holds for all $c>0$. 
Thus if $E\left|X_{11}\right|^{q}=\infty$ for some $q \in(2,4)$ then $S_{n}$ grows at a faster rate than $\sqrt{n \log n}$. The behaviour of $S_{n}$ when $E\left|X_{11}\right|^{q}<\infty$ for all $q<4$, but $E X_{11}^{4}=\infty$ is an open problem. Using Theorem 1 of Rubin and Sethuraman [5] we have that (6) holds in this case, so at least we know that $P\left(\lim \sup _{n \rightarrow \infty}\left(S_{n} / \sqrt{2 n \log n}\right) \geqslant 1\right)=1$.

Finally, a simple application of Theorem 1 of Rubin and Sethuraman [5] gives the following result.

THEOREM 3. Let $\left\{X_{n k}\right\}$ be an array of independent, zero mean random variables such that for each $n, X_{n 1}, X_{n 2}, \cdots$ are identically distributed. If $\operatorname{Var}\left(X_{n 1}\right)=\sigma_{n}^{2}$ and for some $\delta>0, \sup _{n} E\left|X_{n 1}\right|^{4+\delta}<\infty$ then

$$
P\left(\underset{n \rightarrow \infty}{\limsup } S_{n} /\left(\sigma_{n} \sqrt{2 n \log n}\right)=1\right)=1
$$

\section{REFERENCES}

[1] P. Hartman and A. Wintner, 'On the law of the iterated logarithm', Amer. J. Math. 63 (1941), 169-176.

[2] T.C. Hu, F. Moricz and R.L. Taylor, 'Strong laws of large numbers for arrays of rowwise independent random variables', Acta Math. Hung. 54 (1989), 153-162.

[3] A.N. Kolmogorov, 'Sur la loi forte des grandes nombres', Acad. Sci. Paris 191 (1930), 910-912.

[4] R. Michel, 'Results on probabilities of moderate deviations', Ann. Probab. 2 (1974), 349-353.

[5] H. Rubin and J. Sethuraman, 'Probabilities of moderate deviations', Sankhya A 27 (1965), 325-346.

Department of Mathematics

National Tsing-Hua University

Hsinchu

Taiwan 30043
School of Mathematics and Statistics

University of Sydney

New South Wales 2006

Australia 